\title{
HR Management Practices and HR Performance: The Role of Procedural Justice. An Empirical Investigation of Automotive Industry Companies in Morocco
}

\author{
Hamid Latif, PhD student \\ Mohammed Faridi, Professor \\ Jaouad Rharzouz, Professor \\ Hassan First University Settat, Morocco
}

Doi:10.19044/esj.2019.v15n31p81～URL:http://dx.doi.org/10.19044/esj.2019.v15n31p81

\begin{abstract}
Given the centrality of human capital within organizations, many scientific works have focused on this issue. These studies focus in particular on analyzing the link between HRM practices and corporate performance. In large part, the results of previous studies confirm this relationship, while the modalities of influence remain to bediscovered. In Morocco, very few publications have addressed this issue. Thus, our research aims to understand the interaction between HRM practices, procedural justice, and HR performance, using the automotive industry as aground for empirical investigation. To achieve this, we administered a questionnaire to the HR managers of these companies. Based on 65 responsesobtained, the results indicate that incentives and work organization practices indirectly influence HR performance level, while procedural justice plays the intermediary role between the two variables.
\end{abstract}

Keywords: HRM Practices, Procedural Justice, HR Performance

\section{Introduction}

La recherche en Sciences de gestion s'est fortement intéressée à la GRH), en mettant en évidence la pertinence de la problématique d'étude de la contribution de celle-ci à la performance des entreprises. Un ensemble de chercheurs s'accordent sur l'idée selon laquelle certaines pratiques de GRH contribuent à la performance des entreprises. En revanche, les modalités de cette contribution se différent d'une étude à une autre.

Afin de contribuer à comprendre cette relation (GRH-Performance), cette recherche vient pour montrer dans quelle mesure les pratiques de GRH 
sont susceptibles d'exercer une influence sur la performance RH, en s'interrogeant sur la place de la justice procédurale.

Au regard des perspectivesélaborées par Delery et Doty (1996), la perspective universaliste a conséquemment été privilégiée dans le cadre de cette recherche. Une perspective qui tire ses fondements, directement ou indirectement de la théorie des ressources et celle du capital humain pour expliquer le lien entre la GRH et la performance (M. Arcand, Bayad, et Fabi, 2002). Nous nous focalisons sur le secteur de l'industrie automobile comme terrain d'investigation empirique. Un secteur qui constitue une composante principale du Plan d'Accélération Industrielle (PAI ${ }^{5}$ ) 2014-2020 pour sa forte valeur ajoutée et où on assiste à un réel processus de maturation et de développement des pratiques de GRH.

Sur la base de 65 réponses obtenues, une purification des échelles de mesure a été entreprise en mobilisant l'analyse factorielle exploratoire en composantes principales. Ensuite, nous avons opté pour un modèle à équations structurelles sous l'approche PLS, afin de tester le modèle ainsi que les hypothèses de recherche.

\section{Cadre Conceptuel Et Hypotheses}

Selon la revue de la littérature, des académiciens accordent un intérêt grandissant à l'étude du lien entre les pratiques de GRH et la performance (Paré et Tremblay, 2007; Piasecki, 2017). Au Maroc peu de chercheurs se sont intéressés à cette problématique (El Adraoui, 2015).

\section{Pratiques de Gestion des Ressources Humaines}

Longtemps définie comme une activité de support aux autres fonctions de l'entreprise, la GRH représente actuellement un outil stratégique qui permet à une entreprise de se démarquer de ses concurrents (Beker et al. 1997, cité par Chrétien, Lise, Guy Arcand, Geneviève Tellier, et Michel Arcand, 2005). C'est à partir des années 1980, que la GRH a passé d'un rôle purement administratif à un rôle de partenaire stratégique, avec pour objectif l'amélioration de la performance de l'entreprise (Peretti, 2017).

La GRH est définie comme étant « un ensemble d'activités qui ont pout but de fournir à l'entreprise les ressources humaines dont elle a besoin pour atteindre en temps voulu les objectifs qu'elle s'est fixés » (Pigeyre, 2006). Afin d'étudier le lien GRH-Performance, il faut s'intéresser aux pratiques de GRH (Le Louarn, 2006). Audet, Michel, Laurent Bélanger, Richard J. Long, et Bernard Galambaud (1994) précisent que l'expression "pratiques de 
gestion" «sous-entend un ensemble de "manières de faire" de tâches, d'activités propres à chaque entreprise ou un ensemble d'entreprises qui les appliquent ». En ce qui concerne les pratiques en matière de GRH, il s'agit des «outils par lesquels les entreprises peuvent influencer et façonner les compétences, les attitudes et les comportements des individus pour l'exécution du travail et ainsi l'atteinte des objectifs de l'entreprise » (Chen et Huang, 2009 cité par Aliouat et Besbes (2013). Elles sont considérées par Pigeyre (2006) comme la partie «visible » de la GRH : recruter, évaluer, former, etc (Pigeyre, 2006). Les pratiques de GRH correspondent aux façons particulières dont les activités de GRH (recrutement, organisation du travail, formation, évaluation du rendement, etc.) sont accomplies (Rojot et Le Flanchec, 2004 ; Guérin et Wils, 1990;Guérin, G., Wils, T., \& Lemire, L, 1997 ; Carrière et Barrette, 2005). Les résultats de plusieurs recherches suggèrent globalement que les pratiques de GRH peuvent devenir une source de la performance (Ndao,2012;El Adraoui, 2015 ; Pfeffer, 1994 ; Pfeffer, Hatano et Santalainen, 1995 ; Huselid, Jackson et Schuler, 1997).

$\mathrm{Au}$ Maroc, la fonction RH devient, aujourd'hui, de plus en plus formalisée. Elle devient progressivement une réelle GRH effectuée par un spécialiste. Avec ce développent, la fonction RH s'insère dans une vision stratégique. Selon les résultats d'une enquête réalisée en 2012 par le cabinet DIORH, les pratiques de GRH ont connu un développement important, et ce avec la prise en compte des ressources humaines comme variable clé dans la stratégie de l'entreprise, et non plus comme un facteur générateur de coût.

\section{Performance RH}

Depuis quelques années, les chercheurs en GRH se sont intéressés à la problématique de la performance, qui représente le centre d'intérêt des chercheurs en sciences de gestion, pour démontrer le rôle de la GRH dans la performance d'entreprise (Pottiez, 2011).

Un concept qui figure parmi les notions les plus abstraites et floues de la littérature académique, la performance peut être employée pour signifier différentes notions, sans pour autant qu'une définition précise ne fasse l'unanimité.

Au $15^{\text {ème }}$ siècle, il apparaît en anglais avec « to perform » dont vient le mot de performance. Il signifie à la fois accomplissement d'un processus, d'une tâche avec les résultats qui en découlent et le succès que l'on peut y attribuer. Après, le mot performance a été utilisé et ce à partir du $19^{\text {ème }}$ siècle, pour désigner l'ensemble des résultats obtenus par un cheval de course sur les hippodromes, et par extension, le succès remporté dans cette course. Ce terme s'étend ensuite vers la fin du $19^{\text {ème }}$ siècle, pour désigner les résultats et l'exploit sportif d'un athlète. 
Son sens évolua au cours du 20ème siècle ; il indiquait de manière chiffrée les possibilités d'une machine et désignait par extension un rendement exceptionnel. Pesqueux (2004) a conclu par dire que « le mot est en quelque sorte "attrape tout" dans la mesure où il comprend à la fois l'idée d'action (performing) et d'état (performance comme étape franchise) ». D'après Des Horts (2004), c'est avec l'intérêt des chercheurs à comprendre le lien GRHPerformance que la performance RH et sa mesure ont été explicitement abordées. L'adjonction du terme performance à celui de ressources humaines peut paraître surprenante (Gilbert et Charpentier, 2004). Pendant que le premier renvoie à des idées de mesure, de quantification et d'évaluation et le second est généralement associé à l'Homme et à sa complexité (Louart, 1996). La performance RH est un concept multidimensionnel et subjectif (Pottiez, 2011). Comme disaient Gilbert et Charpentier (2004), l'évaluation et la mesure de la performance RH est une problématique universelle, mais les réponses apportées sont variées du fait de la multitude de contextes dans lesquels évoluent les entreprises.

Pour notre étude, nous allons approcher la performance RH à la dimension valeur RH du modèle de Morin, Estelle M., André Savoie, et Guy Beaudin.(1994). Un modèle qui nous permettra d'opérationnaliser facilement le concept de la performance RH grâce à la présence de nombreux indicateurs (Boulianne, 2002). Naro dans son article de 2006 propose un ensemble d'indicateur permettant de mesurer la dimension RH de l'entreprises, dont le taux de turnover, le taux d'absentéisme, le taux de grève, la satisfaction des salariés...). La performance $\mathrm{RH}$ est généralement définie à travers ses indicateurs. Dans ce sens, Morin, Estelle M., André Savoie, et Guy Beaudin(1994) et Naro (2006) proposent plusieurs indicateurs pour la mesurer : «effort de formation, mobilité des employés, dépense consacrée au recrutement et à la formation, nombre de jours de formation, Nombre de jours perdus à cause d'un arrêt de travail, nombre de griefs reçus, nombre d'accidents de travail, taux d'absentéisme, taux de rotation des employés, taux de participation aux activités sociales, taux de maladie, revenus par employé, bénéfice net avant impôt par employé, Taux d'encadrement, Degré de satisfaction, implication des employés, engagement des employés, ... ».

\section{Le lien GRH-Performance et modèle conceptuel de la recherche}

Arcand (2001), lors de l'inventaire des divers écrits et recherches, qui ont tenté d'étudier et d'expliquer la relation GRH-Performance, a constaté que cette relation est loin de constituer l'unanimité parmi les chercheurs et théoriciens de la GRH. S'intéressant aux recherches portant sur cette problématique, Delery et Doty (1996), dans un travail de synthèse et de clarification conceptuelle, distinguent trois approches différentes, à savoir : la perspective universaliste qui ne tient compte que de l'implantation de pratiques 
exemplaires de GRH pour influencer la performance ; la perspective de la contingence, pour sa part tient compte de la stratégie de l'entreprise pour choisir les pratiques de GRH à instaurer pour influencer la performance ; et la perspective configurationnelle qui stipule que les pratiques de GRH doivent avoir une grande consistance interne en lien avec la stratégie de l'entreprise pour influencer la performance (Ndayirata, Belghiti-Mahut, et Briole, 2018; AllaniSoltan, Arcand, et Bayad, 2003; Gagnon et Arcand, 2012; Simen, 2017). Malgré les résultats de quelques recherches, le lien pratiques de GRHPerformance RH (attitude-comportement) reste nébuleux (Paré \& Tremblay, 2007). Pour cette raison et d'autres, plusieurs chercheurs tels que Paré et Tremblay (2007) et Meyer \& Smith (2000) soulignent qu'il est important de comprendre comment ces pratiques de GRH influencent les indicateurs de la performance RH. Ce lien, comme disait Aït Razouk (2007), peut paraitre évident pour certains chercheurs en management. Mais, il est de plus en plus délicat de le démontrer scientifiquement. De ce fait, plusieurs auteurs (Becker et Huselid,2006;Youndt et Snell, 2004 ; Wright, Gardner et Moynihan, 2003) ont fait appel à la communauté scientifique afin que les futures recherches en matière de lien entre les pratiques de GRH et la performance portent davantage sur le mécanisme intermédiaire qui s'opère entre les variables figurant aux extrémités de la relation. Dans ce cadre, plusieurs études ont proposé une panoplie de variables dont figure la justice procédurale.Une varibaleconsidérée comme un facteur prédictif de la performance (Reb, Jochen, Barry M. Goldman, Laura J. Kray, et RussellCropanzano, 2006). Il s'agit d'un des éléments-clés permettant d'expliquer comment certaines pratiques de GRH influencent les attitudes et les comportements en milieu de travail (Cohen-Charash et Spector,2001;Colquitt, 2001; Cropanzano et Greenberg, 1997; Folger et Konovsky, 1989).

En résumé, les résultats de différentes recherches et méta-analyses confirment que la performance RH à travers ses composantes (la satisfaction, l'absentéisme, le turnover etc) est influencé par les jugements globaux de justice déterminée bien souvent par les politiques et pratiques organisationnelles (Ambrose et Schminke,2009; Duguay, 2006; Kivimäki, Mika, JussiVahtera, Marko Elovainio, Marianna Virtanen, et Johannes Siegrist, 2007; Simard, Doucet, et Bernard 2005 ; Paré \& Tremblay, 2007). Ces liens importants soulignent l'importance de ne pas négliger ce concept clé. En s'intéressant à ce qui nous aide à déterminer les perceptions de justice, notamment au sein des pratiques organisationnelles et caractéristiques de travail, nous pourrons faire de la justice un levier d'actions pour booster la performance RH (Piasecki, 2017). Dans leur article, Simard, Doucet, et Bernard (2005) démontrent clairement le rôle médiateur de la justice dans la relation entre les pratiques de GRH et certains indicateurs de la performance RH. Lambert, E. G., Hogan, N. L., Jiang, S., et Elechi (2010) indiquent que la 
justice procédurale devrait être priorisée avant toutes les autres dimensions de la justice organisationnelle (Renaud, Tremblay, et Morin, 2014). Elle est considérée ainsi comme un déterminant majeur des résultats, attitudes et comportements envers l’organisation dans sa globalité (Masterson Suzanne S, Kyle Lewis, Barry M. Goldman and M. Susan Taylor, 2000; Piasecki, 2017). Cropanzano et Byrne (2000) ont trouvé aussi que la justice procédurale était le meilleur prédicteur des variables au niveau organisationnel. La métaanalyse de Cohen-Charash et Spector (2001), suggère que la justice procédurale est fortement liée à la performance RH. Les résultats d'une recherche faite en 2005 par Simard, Drolet et Bernard, démontrent clairement le rôle médiateur de la justice dans la relation entre pratiques de GRH et performance $\mathrm{RH}$. Une variable qui s 'inscrit très largement dans la perspective de la théorie de l'échange social (Blau, 1964).

\section{La Justice Procédurale}

A partir des années 80, certains chercheurs se sont intéressés aux processus menant à l'attribution des ressources, en introduisant le concept de la justice procédurale. Thibaut et Walker (1975) ont introduit le concept de justice procédurale à travers le contexte de la prise de décision juridique. Ils indiquent que pour qu'une procédure juridique soit jugée juste, il est nécessaire d'avoir un sentiment de contrôle sur le processus de décision (donner son opinion, fournir une argumentation...) ou sur la décision ellemême, à savoir la possibilité d'influencer le résultat décisionnel (Piasecki, 2017). La justice procédurale a un rôle majeur puisqu'elle permet en cas de distribution injuste d'accepter ces résultats peu avantageux et le jugement d'une décision non favorable sera donc plus positif si la procédure est juste. C'est un jugement sur les procédures et les processus mobiliséspour la prise de décision (Brien-Robidoux, Emmanuelle, Camille Labrèche, DavidEmmanuel Hatier, Anne-Marie Paiement, et Mickael Blais,2017 ; Cloutier, 2003 ; Müller \&Djuatio, 2011 ; Ndayirata, 2017 ; Caroline Piasecki, 2017). Paillé (2011) affirme, en se basant sur les travaux de Cropanzano et Mitchell (2005), que la théorie de l'échange social (Blau, 1964), « fournit des arguments théoriques solides pour mieux examiner les comportements en milieu de travail ». Selon cette théorie, les organisations sont considérées comme des lieux de relations sociales mutuelles à long terme entre employés et employeurs (Paré \& Tremblay, 2007). Plusieurs recherches en GRH l'ont effectivement mobilisé comme cadre théorique permettant de prédire les effets des pratiques de GRH sur les attitudes et les comportements des employés au travail (Serge Ndayirata 2018; Gould-Williams et Davies, 2005 ; M Tremblay, J Cloutier, G Simard; Cheng, 2014).

La revue de littérature que nous venons de présenter permet de croire à l'existence d'un lien indirect entre les pratiques en matière de gestion des 
ressources humaines et la performance RH et que la justice procéduralejout un rôle intérmidiaire. Nous pouvons, donc proposer le modèle suivant :

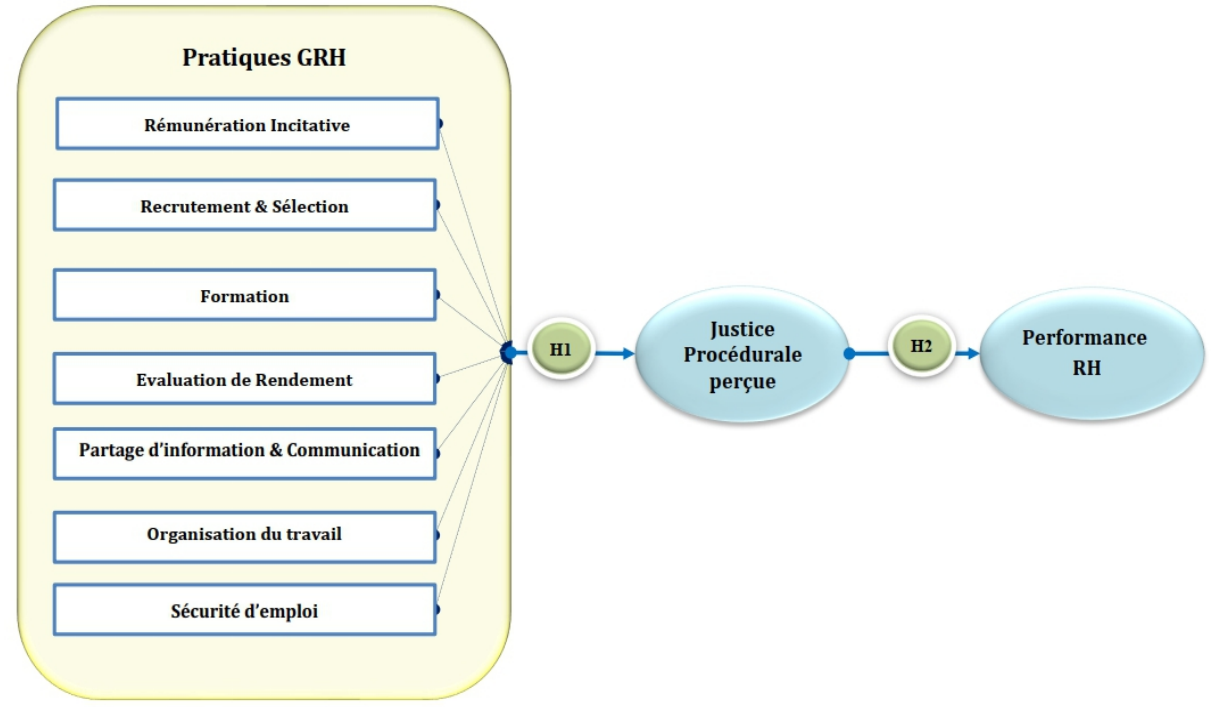

Figure 1. Modèle conceptuel de recherche

Ce modèle émet des questionnements et suppose des relations entre des variables. Rappelons que nous tentons d'identifier si les pratiques de GRH (variables indépendantes), recensées au sein des entreprises participantes, peuvent améliorer la performance RH (variable dépendante) en s'interrogeant sur le rôle de la justice procédurale.

\section{Methodologie De Recherche}

Après avoir élaboré un modèle conceptuel permettant de questionner le processus par lequel les pratiques de GRH influencent la perception de la justice procédurale et la performance $\mathrm{RH}$, nous avons identifié les différents items servant à mesurer les différentes variables. Nous tentons par cette recherche corrélationnelle d'établir une relation de cause à effet entre ces variables.

La phase d'opérationnalisation des construits a permis d'élaborer un questionnaire de recherche destiné aux entreprises de l'industrie automobile marocaine. Cette recherche s'appuie sur une étude quantitative en deux temps. En premier lieu, une étude quantitative exploratoire afin de purifier les échelles de mesure des différentes variables (analyse en composantes principales et analyse de la fiabilité), puis, une approche quantitative confirmatoire via les méthodes des équations structurelles sous l'approche PLS, afin de valider les échelles de mesure, les hypothèses et le modèle de recherche. Notre recherche s'inscrit dans une posture épistémologique 
positiviste où nous tenterons de valider nos hypothèses de recherche présentées au préalable (approche hypothético-déductive).

\section{Terrain d'enquête et échantillon}

Nous avons choisi le secteur de l'industrie automobile, un secteur considéré stratégique pour l'économie marocaine (Boubker \& Chafik, 2016), comme terrain empirique de notre recherche. « Grâce à la stratégie émergente adoptée par le Maroc, l'industrie automobile représente aujourd'hui un secteur à forte valeur ajoutée, elle a enregistré durant ces dernières années une performance à l'export très remarquable, à travers le développement de l'activité du câblage et l'essor du segment de la construction automobile » (Boubker, 2017. P. 133). C'est un secteur où la GRH est plus formalisée.

Cohérente avec notre problématique, notre stratégie de recherche se fait par le biais d'une enquête par questionnaire. Plus particulièrement, elle porte sur 200entreprises de l'industrie automobile. Comme critères d'éligibilité à la recherche, nous n'avons retenu que les entreprises ayant plus de 100 employés et qui ont un service ou département dédié à la GRH. Ce choix méthodologique nous a permis de ne retenir que les entreprises les plus importantes, accroissant donc la probabilité de retrouver des pratiques de GRH formelles.

Le faible nombre d'entreprises du secteur de l'industrie automobile au Maroc ne nous a pas permis de faire un tirage aléatoire des entreprises que nous voulions étudier. Nous avons donc considéré toutes les entreprises répondants à nos critères de sélection et nous les avons inclus dans notre enquête. Nous avons bénéficié de l'appui d'un ensemble d'organisations telle que, l'AMICA ${ }^{6}$, de la fédération de l'automobile $\left(\mathrm{CGEM}^{7}\right)$ et l'ANAPEC $^{8}$ afin d'obtenir toutes les informations et toutes les facilités nécessaires à notre recherche. Dans le cadre de cette étude, une centaine d'entreprises ont été sollicitées et nous avons obtenu 65 réponses.

\section{Mesures des variables}

En se basant sur la littérature, il nous a été possible de proposer un modèle de base qui permettrait d'évaluer la relation entre les variables de notre problématique. Selon des perspectives très diverses, plusieurs chercheurs se sont intéressés à l'analyse du positionnement de la GRH et de la justice procédurale dans la problématique de la performance $\mathrm{RH}$. Les différentes variables de notre modèle de recherche ont été adoptées dans différents contextes. Dans ce sens, afin de proposer un échantillon d'items adapté à notre

\footnotetext{
${ }^{6} \mathrm{~L}$ 'Association Marocaine des Industriels et Constructeurs Automobiles

${ }^{7}$ Confédération générale des entreprises du Maroc

${ }^{8}$ Agence nationale de promotion d'emplois et des compétences
} 
contexte de recherche, nous avons opté pour les items les plus mobilisés au niveau de la littérature, en respectant les deux premières phases du paradigme préconisé par (Churchill, 1979). Nous avons procédé à une analyse factorielle exploratoire, afin de mettre en évidence les différentes dimensions servant à mesurer nos variables étudiées. Les pratiques de GRH, La justice procédurale et la performance RH ont été mesurées sur une échelle de Likert en sept points. Les échelles qui ont été utilisées pour les mesurer sont décrites dans ce qui suit.

\section{Les pratiques de GRH}

Parmi les principales recherches qui nous ont inspiré, nous pouvons citer Arcand (2001); G. Arcand (2006); Zgoulli-Swalhi(2014); Chrétien, Lise, Guy Arcand, Geneviève Tellier, et Michel Arcand,(2005); Boselie, Dietz, et Boon (2005); Laaboubi(2012); Wu et Chaturvedi(2009); Duguay (2006); Ndao (2012); Paré et Tremblay (2007) ; El adraoui(2015). Ces recherches consultées utilisent un large éventail de pratiques de GRH. Pour réaliser cette étude, nous retiendrons, pour des considérations de validité, les pratiques les plus communément utilisées et reconnues par la littérature scientifique et qui caractérisent notre terrain d'enquête. Arcand (2001), Arcand (2006), Laaboubi (2012) et Ndao (2012) font part des pratiques de GRH largement utilisées. À la lumière des différentes lectures sur le sujet et sous le conseil de plusieurs experts (pré-test), nous avons sélectionné sept groupes de pratiques de GRH qui convenaient le mieux à l'étude (La rémunération incitative, Recrutement et Sélection, Formation, Evaluation du rendement, Partage d'information et communication, Organisation du travail et la Sécurité d'emploi).

Comme pour la majorité des recherches en GRH-performance, c'est par le biais d'un questionnaire que nous avons mesuré le niveau d'implantation de ces pratiques. Alors que la plupart des études effectuées mesurent la présence ou l'absence d'une pratique au sein d'une entreprise (Wall \& Wood, 2005), nous avons choisi une mesure, développée par Huselid (1995), qui permet d'évaluer le degré d'implantation d'une pratique de GRH auprès des employés. Pour la majorité des pratiques, l'implantation a été mesurée à partir d'un seul et même indicateur, soit le pourcentage d'employés touchés par l'utilisation d'une pratique ( 1 = pas de tout implantée auprès de $0 \%$ des employés et 7 = fortement implantée auprès de $100 \%$ des employés).

\section{La performance $\mathbf{R H}$}

Notre questionnaire mesure la performance $\mathrm{RH}$ des entreprises par un ensemble d'indicateurs les plus utilisés dans la littérature (Morin, Estelle M., André Savoie, et Guy Beaudin, 1994; Naro, 2006) et caractérisent notre terrain d'enquête: Taux de roulement, Taux d'absentéisme, Taux de maladie, Nombre d'accidents de travail, Climat social, Productivité, Taux 
d'encadrement, Mobilité des employés, Satisfaction des employés, Effort de formation (Taïeb 2016 ; El adraoui, 2015 ; Imbert 2007; Naro, 2006 ;Morin, Estelle M., André Savoie, et Guy Beaudin, 1994).

De façon plus précise, pour mesurer cette variable, nous avons demandé aux répondants de nous indiquer, selon l'échelle de Likert en sept points, leur degré de satisfaction par rapport aux différents indicateurs de performance RHdans leurs entreprises.

\section{La justice procédurale}

La présente étude s'intéresse plus particulièrement à la justice procédurale, car elle est des types de justice formellement mises en place dans les entreprises (Brien-Robidoux, Emmanuelle, Camille Labrèche, DavidEmmanuel Hatier, Anne-Marie Paiement, et Mickael Blais, 2017 ; Tyler et Blader, 2003). Afin d'accroître la qualité de l'information, l'une des précautions prise est la triangulation des informations relatives à cette variable. Nous avons demandé qu'il y ait trois répondants par entreprise pour évaluer les perceptions de la justice procédurale (Gestionnaire RH, Délégué du personnel et représentant syndical).

Pour la mesurer, on s’inspirant des travaux de Müller et Djuatio(2011); Gangloff, Finkelstein, et Holmick(2017) et Salhi(2016), nous avons demandé aux participants de nous indiquer, selon l'échelle de Likert en sept points, leur degré d'accord ou de désaccord par rapport aux items constituant cette variable ( $1=$ Fortement en désaccord et $7=$ Tout à fait d'accord).

\section{Administration du questionnaire}

Afin de concevoir un outil adéquat et répondant aux objectifs de notre enquête, deux séries de pré-tests ont été effectuées afin d'assurer la fiabilité du questionnaire et sa compréhensibilité par les participants. La première s'est faite avec des enseignants spécialisés. La seconde a été faite avec un groupe réduit de professionnels appartenant aux entreprises de l'échantillon.

Les pratiques de GRH, les items de la justice procédurale et les indicateurs de performance RH identifiés dans notre questionnaire sont donc représentatifs de ceux en usage dans les entreprises de notre échantillon.

Le questionnaire a été administré directement auprès des gestionnaires RH et représentant du personnel dans les entreprises participantes. Les questionnaires étaient recueillis immédiatement. Cette méthode nous a permis d'augmenter le nombre de questionnaires exploitables dans la phase d'analyse des données. 


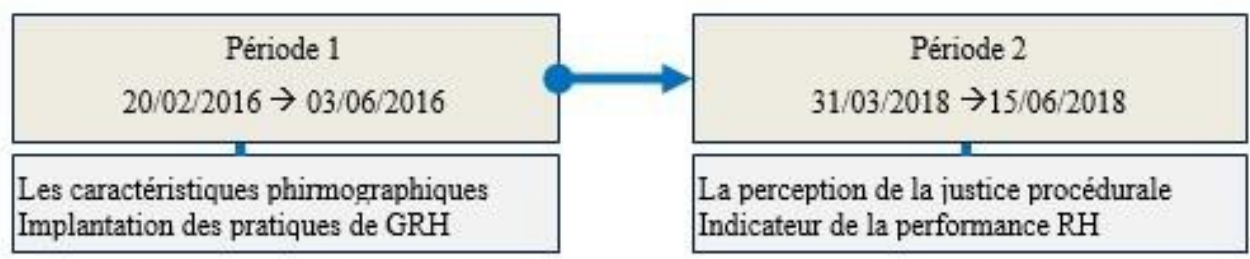

\section{Resultats Et Discussions}

Les résultats obtenus indiquent que nos données sont métriques et factorisables. Les tests de $\mathrm{KMO}^{9}$ et de sphéricité de Bartlett sont positifs etpermettent de vérifier la capacité de nos données à être factorisées. Une purification des échelles de mesure des variables de cette recherche a été faite. Pour les pratiques de GRH : le tableau ci-dessous synthétise les résultats obtenus en phase de purification de l'échelle de mesure des pratiques GRH. L'alpha de Cronbach pour les cinq dimensions respecte les normes scientifiques. De même, la qualité de représentation et la contribution factorielle sont supérieures à 0,5 .

\begin{tabular}{|c|c|c|c|c|c|c|c|c|c|c|}
\hline \multirow{2}{*}{ Dimensions } & \multirow{2}{*}{ Items } & \multirow{2}{*}{$\begin{array}{c}\text { Nbre } \\
\text { d'items }\end{array}$} & \multirow{2}{*}{\begin{tabular}{c|} 
Qualité de \\
représentation
\end{tabular}} & \multicolumn{5}{|c|}{ Contribution Factorielle } & \multicolumn{2}{|c|}{$\alpha$ de Crombach } \\
\hline & & & & Communication & O.T & R.I & S.T & Formation D & Dimension & \multirow{20}{*}{0,918} \\
\hline \multirow{6}{*}{$\begin{array}{c}\text { Communication } \\
\& \\
\text { Partage } \\
\text { d'information }\end{array}$} & PGRHCommPartInfoEse & \multirow{6}{*}{6} & 980 & 961 & & & & & \multirow{6}{*}{0,990} & \\
\hline & PGRHCommPartInfClts & &, 985 &, 960 & & & & & & \\
\hline & PGRHCommPartInfConc & & 972 & ,959 & & & & & & \\
\hline & PGRHCommCultureEse & & 961 & ,958 & & & & & & \\
\hline & PGRHCommPartInfAugSal & & 981 &, 953 & & & & & & \\
\hline & PGRHCommPartInfStra & & 869 & ,908 & & & & & & \\
\hline \multirow{4}{*}{$\begin{array}{c}\text { Organisation de } \\
\text { travail }\end{array}$} & PGRHOrgaTvEnrichTask & \multirow{4}{*}{4} & 951 & & ,929 & & & & \multirow{4}{*}{0,961} & \\
\hline & PGRHOrgaTvElargTask & & 904 & & ,895 & & & & & \\
\hline & PGRHOrgaTvRésolProb & & 921 & &, 890 & & & & & \\
\hline & PGRHOrgaTvPolyval & & 840 & & 869 & & & & & \\
\hline \multirow{4}{*}{$\begin{array}{l}\text { Rémunération } \\
\text { Incitative }\end{array}$} & PGRHRémIncPrixSymb & \multirow{4}{*}{4} & 932 & & & ,944 & & & \multirow{4}{*}{0,961} & \\
\hline & PGRHRémIncRécomNM & & 877 & & & ,918 & & & & \\
\hline & PGRHRémIncAvatNM & & 926 & & & 895 & & & & \\
\hline & PGRHRémIncPrIndi & & ,890 & & & 869 & & & & \\
\hline \multirow{3}{*}{ Sécurité de travail } & PGRHSécuTvProetcEmp & \multirow{3}{*}{3} & 881 & & & & ,923 & & \multirow{3}{*}{0,871} & \\
\hline & PGRHSécuTvSauvOutTv & & 842 & & & & ,912 & & & \\
\hline & PGRHSécuTvSalRég & &, 755 & & & & ,796 & & & \\
\hline \multirow{2}{*}{ Formation } & PGRHFormProGénAdap & \multirow{2}{*}{2} & 990 & & & & & ,943 & \multirow{2}{*}{0,993} & \\
\hline & PGRHFormProSpéAdap & & 992 & & & & &, 937 & & \\
\hline \multicolumn{4}{|c|}{ Valeur propre } & 8,120 & 3,561 & 2,653 & 1,924 & 1,190 & \multirow{4}{*}{\multicolumn{2}{|c|}{$\begin{array}{c}\text { KMO }=0,746 \\
P=0.000 \\
N=65\end{array}$}} \\
\hline & Test de Bartlett : Signific & atif & & \multicolumn{5}{|c|}{ Varimax } & & \\
\hline \multirow{2}{*}{\multicolumn{4}{|c|}{ Variance expliquée en \% }} & 30,448 & $19,171 \%$ & $18,863 \%$ & $13,086 \%$ & $10,264 \%$ & & \\
\hline & & & & & 91 , & $332 \%$ & & & & \\
\hline
\end{tabular}

Tableau 1. Résultats de purification des pratiques $\mathrm{RH}$

Les analyses effectuées lors de la phase de purification et de test ont permis de valider l'échelle de mesure des pratiques de GRH à :

${ }^{9}$ Appelé aussi le test MSA (Measure of Sampling Adequacy) 


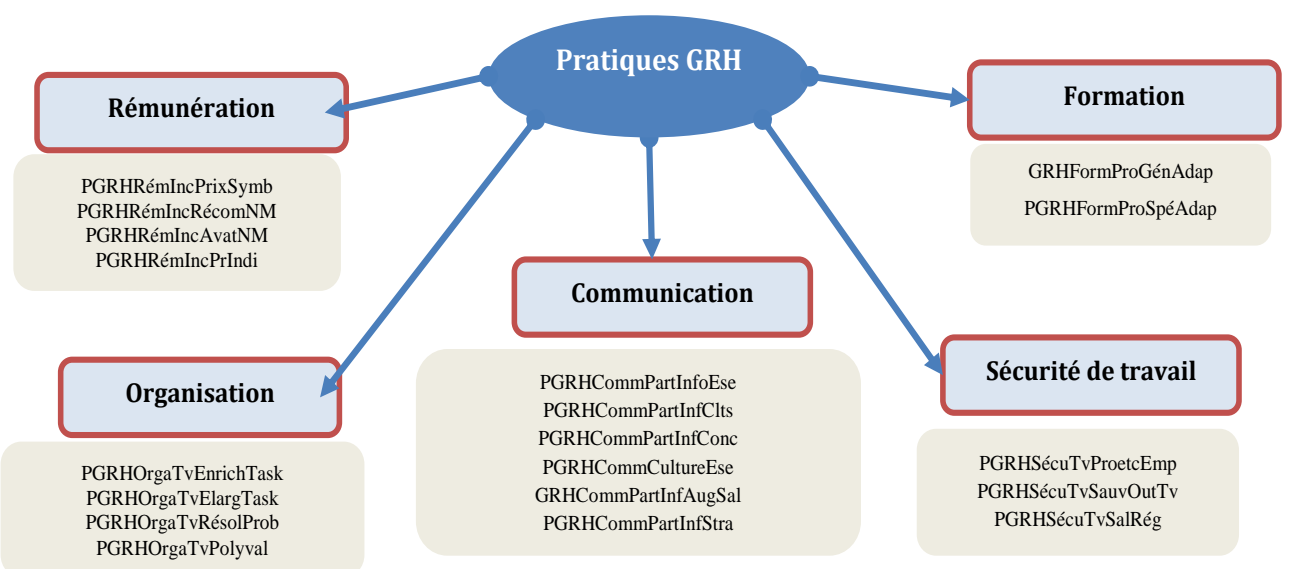

Figure 2. Liste de dimensions conservées pour la varibale pratiques de GRH

Suite aux itérations de l'analyse factorielle, les énoncés qui sont mal représentés ont été écartés. En résumé, nous avons obtenu un résultat avec une échelle réduite à cinq dimensions et 19 items.

Pour la justice procédurale et lors de la phase d'opérationnalisation des construits, nous avons identifié 8 items servant à la mesurer. L'échelle de mesure de la justice procédurale est fiable avec une valeur de l'alpha de Cronbach qui respecte les normes en sciences de gestion.

De même, la qualité de représentation et la contribution factorielle des différents items sont supérieures à 0,5. En somme, la variable justice procédurale est une variable unidimensionnelle mesurée via trois items :

$\bigotimes$ Les salariés peuvent donner leurs opinions sur les procédures de l'entreprise.

$\bigotimes$ L'entreprise traite rapidement les demandes de formation des salariés.

$\bigotimes$ L'entreprise laisse toujours aux salariés le temps d'exprimer par rapport à leur travail.

Le tableau ci-dessous synthétise les résultats obtenus en phase de purification de l'échelle de mesure de la justice procédurale :

\begin{tabular}{|c|c|c|c|c|}
\hline Liste des items & Nbre d'items & $\begin{array}{l}\text { Qualité de } \\
\text { représentation }\end{array}$ & $\begin{array}{c}\text { Contribution } \\
\text { Factorielle }\end{array}$ & $\alpha$ Crombach \\
\hline JustProcOpin & \multirow{3}{*}{3} & ,855 & ,925 & \multirow{3}{*}{0,917} \\
\hline $\begin{array}{c}\text { JustProcDemFor } \\
\mathrm{m}\end{array}$ & & 839 & ,916 & \\
\hline JustProcExprTv & & 892 & ,945 & \\
\hline \multicolumn{3}{|c|}{ Valeur propre } & 2,587 & \multirow{3}{*}{$\begin{array}{c}\mathrm{KMO}=0,7 \\
50 \\
\mathrm{P}=0.000 \\
\mathrm{~N}=65\end{array}$} \\
\hline \multicolumn{3}{|c|}{ Test de Bartlett : Significatif } & Varimax & \\
\hline \multicolumn{3}{|c|}{ Variance expliquée en $\%$} & 86,22 & \\
\hline
\end{tabular}

Tableau 2. Résultats de purification de l'échelle de la justice procédurale 
Pour la performance $\mathbf{R H}$, le tableau ci-dessous synthétise les résultats obtenus en phase de purification de son échelle de mesure.

\begin{tabular}{|c|c|c|c|c|}
\hline Liste des items & $\begin{array}{c}\text { Nbre } \\
\text { d'items }\end{array}$ & $\begin{array}{c}\text { Qualité de } \\
\text { représentation }\end{array}$ & $\begin{array}{c}\text { Contribution } \\
\text { Factorielle }\end{array}$ & $\alpha$ Crombach \\
\hline PerfoRHTAbs & \multirow{3}{*}{3} & ,837 & ,915 & \multirow{3}{*}{0,872} \\
\hline PerfoRHProduc & & ,857 & ,926 & \\
\hline PerfoRHSatisf & & ,707 & ,841 & \\
\hline \multicolumn{3}{|c|}{ Valeur propre } & 2,401 & \multirow{3}{*}{$\begin{array}{c}\mathrm{KMO}=0,7 \\
05 \\
\mathrm{P}=0.000 \\
\mathrm{~N}=65\end{array}$} \\
\hline \multicolumn{3}{|c|}{ Test de Bartlett : Significatif } & Varimax & \\
\hline \multicolumn{3}{|c|}{ Variance expliquée en \% } & 80,038 & \\
\hline
\end{tabular}

Tableau 3. Résultats de purification de l'échelle de mesure de la performance RH

Sur la base des analyses effectuées nous avons validé l'échelle de mesure de la performance RH. Ainsi, les résultats suggèrent que cette variable latente est mesurée via trois indicateurs. Le premier renvoi au taux d'absentéisme. Alors que le deuxième critère est lié à la productivité. Le dernier indicateur renvoie à la satisfaction des employés (clients internes).

$\bigotimes$ Tauxd'absentéisme,

冈 Productivité,

$\bigotimes \quad$ La satisfaction des employés.

Suite à l'analyse factorielle exploratoire en composantes principales, nous avons étudié les structures factorielles des trois variables de notre modèle de recherche.

\begin{tabular}{|c|c|c|c|c|c|}
\hline \multirow{2}{*}{\multicolumn{2}{|c|}{ Variables \& Dimensions }} & \multirow{3}{*}{ Listes des items } & \multirow{3}{*}{$\frac{\text { Codes }}{\text { PGRHCommPartinfoEse }}$} & \multicolumn{2}{|c|}{ A de Cronbach } \\
\hline & & & & Dimensions & Variable \\
\hline \multirow{19}{*}{ 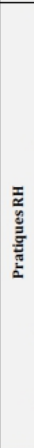 } & \multirow{6}{*}{$\begin{array}{c}\text { Communication \& } \\
\text { partage } \\
\text { d'informations } \\
\text { ( } 6 \text { items) }\end{array}$} & & & \multirow{6}{*}{0,990} & \multirow{19}{*}{0,918} \\
\hline & & 2. Partager les informations sur les clients & PGRHCommPartInfClts & & \\
\hline & & 3. Partager les informations sur la concurrence & PGRHCommPartInfConc & & \\
\hline & & 4. Actions de renforcement de la culture d'entreprise & PGRHCommCultureEse & & \\
\hline & & 5. Partager les informations sur les critères de détermination et d'augmentation du salaire & PGRHCommPartinfAugSal & & \\
\hline & & 6. Partager les informations sur les stratégies et objectifs de l'entreprise & PGRHCommPartInfStra & & \\
\hline & \multirow{4}{*}{$\begin{array}{l}\text { Organisation du } \\
\text { travail } \\
\text { (4 items) }\end{array}$} & 1. Enrichissement des tâches & PGRHOrgaTvEnrichTask & \multirow{4}{*}{0,961} & \\
\hline & & 2. Elargissement des tâches & PGRHOrgaTvElargTask & & \\
\hline & & 3. Groupe de résolution des problèmes & PGRHOrgaTvRésolProb & & \\
\hline & & 4. Développement de la polyvalence & PGRHOrgaTvPolyval & & \\
\hline & \multirow{4}{*}{$\begin{array}{l}\text { Rémunération } \\
\text { Incitative } \\
\text { (4 items) }\end{array}$} & 1. Attribution de prix symboliques & PGRHRémIncPrixSymb & \multirow{4}{*}{0,961} & \\
\hline & & 2. Récompenses non monétaires (voyage, cadeaux...) & PGRHRémIncRécomNM & & \\
\hline & & 3. Avantages non monétaires (voiture...) & PGRHRémIncAvatNM & & \\
\hline & & 4. Primes et bonus individuels & PGRHRémIncPrIndi & & \\
\hline & \multirow{3}{*}{$\begin{array}{l}\text { Sécurité d'emploi } \\
\text { (3 items) }\end{array}$} & 1. S'engager à protéger les emplois & PGRHSécuTvProetcEmp & \multirow{3}{*}{0,871} & \\
\hline & & 2. Sensibilisation pour la sauvegarde de l'outil de production & PGRHSécuTvSauvOutTV & & \\
\hline & & 3. Payement régulier des salaires & PGRHSécuTvSalRég & & \\
\hline & \multirow{2}{*}{$\begin{array}{c}\text { Formation } \\
\text { ( } 2 \text { items) }\end{array}$} & 1. L'utilisation des PGF permettant de rendre les employés plus aptes à répondre aux besoins futurs de l'entreprise & PGRHFormProGénAdap & \multirow{2}{*}{0,993} & \\
\hline & & 2. L'utilisation de PSF permettant de rendre les employés plus aptes à répondre aux exigences de leur poste & PGRHFormProSpéAdap & & \\
\hline \multirow{3}{*}{\multicolumn{2}{|c|}{ Justice Procédurale }} & 1. Les salariés peuvent donner leurs opinions sur les procédures de l'entreprise. & JustProcOpin & \multirow{3}{*}{\multicolumn{2}{|c|}{0,917}} \\
\hline & & 2. L'entreprise traite rapidement les demandes de formation des salariés. & JustProcDemForm & & \\
\hline & & 3. L'entreprise laisse toujours aux salariés le temps d'exprimer par rapport à leur travail. & JustProcExprTv & & \\
\hline \multirow{3}{*}{\multicolumn{2}{|c|}{ Performance RH }} & 1. Taux d'absentéisme & PerfoRHTAbs & \multirow{3}{*}{\multicolumn{2}{|c|}{0,872}} \\
\hline & & 2. Productivité (CA/Effectif) & PerfoRHProduc & & \\
\hline & & 3. La satisfaction des employés & PerfoRHSatisf & & \\
\hline
\end{tabular}

Tableau 4. Synthèse des résultats de la phase de purification des échelles de mesure

Les résultats liés à la phase de validité convergente des différents modèles de mesure des variables latentes de notre recherche indiquent que l'ensemble indices respecte les normes scientifiques, en termes des différents indices liés à la validité convergente. À ce niveau les échelles de mesure des 
différentes variables latentes présentent un niveau de fiabilité suffisant (l'ensemble des valeurs obtenues sont au-delà de 0,7). Avec des contributions factorielles supérieures à 0,7 . De même, l'indice AVE pour l'ensemble des modèles de mesure est au-delà de 0,5. La validité discriminante évaluée suite à l'examen de deux critères; les corrélations entre construits et les contributions croisées, sont satisfaisantes. En somme, d'après les analyses dédiées à la validité convergente et discriminante, nous pouvons confirmer la validité des différents modèles de mesure des variables latentes de notre recherche.

La validation du modèle structurel interne avec PLS se fait moyennant trois indicateurs : l'indice de qualité (GOF), le coefficient de détermination du modèle $\left(\mathrm{R}^{2}\right)$ et l'effet de taille $\mathrm{R}^{2}\left(\mathrm{f}^{2}\right), \mathrm{Q}^{2}$, GoF, Path Coefficient.

\begin{tabular}{|c|c|c|c|c|c|c|c|c|c|}
\hline \multicolumn{5}{|c|}{ Hypothèses } & $\begin{array}{l}\text { Std. Béta } \\
(\beta)\end{array}$ & $T$-value & P-value & $\begin{array}{c}\text { Niveau de } \\
\text { significativité }\end{array}$ & $\begin{array}{l}\text { Statut de } \\
\text { I'hypothèse }\end{array}$ \\
\hline \multirow{5}{*}{ H1 } & H1.1 & Communication & $\rightarrow$ & Justice procédurale & 0,011 & 0,095 & 0,924 & $\begin{array}{c}\text { Non } \\
\text { Significative }\end{array}$ & Infirmée \\
\hline & H1.2 & $\begin{array}{c}\text { Organisation du } \\
\text { travail }\end{array}$ & $\rightarrow$ & Justice procédurale & 0,443 & 3,872 & 0,000 & $\begin{array}{c}* * * \text { Significative à } \\
\mathrm{p}<0.001\end{array}$ & Confirmée \\
\hline & H1.3 & $\begin{array}{l}\text { Rémunération } \\
\text { Incitative }\end{array}$ & $\rightarrow$ & Justice procédurale & 0,266 & 2,303 & 0,021 & $\begin{array}{c}{ }^{*} \text { Significative à } \\
\mathrm{p}<0.05\end{array}$ & Confirmée \\
\hline & H1.4 & Sécurité d'emploi & $\rightarrow$ & Justice procédurale & $-0,210$ & 1,771 & 0,077 & $\begin{array}{c}\text { Non } \\
\text { Significative }\end{array}$ & Infirmée \\
\hline & H1.5 & Formation & $\rightarrow$ & Justice procédurale & $-0,051$ & 0,421 & 0,674 & $\begin{array}{c}\text { Non } \\
\text { Significative }\end{array}$ & Infirmée \\
\hline & Justice procédurale & $\rightarrow$ & Performance RH & $0 ., 350$ & 2,872 & 0,005 & $\begin{array}{c}\text { ** }^{* *} \text { Significative à } \\
\mathrm{p}<0.01\end{array}$ & Confirmée \\
\hline
\end{tabular}

Tableau 5. Tests d'hypothèse de recherche sur la base d'un MES-PLS

Les résultats de la présente recherche ont permis de confirmer trois soushypothèses et d'infirmer trois.

D'après le tableau ci-dessus, nous pouvons conclure que la relation entre les pratiques de GRH liées à la communication et le partage d'informations et la justice procédurale n'est pas significative $(H 1.1: \beta=$ $0,011, \mathrm{t}=0,095, \mathrm{p}=0.924)$. Ainsi, cette première hypothèse est rejetée. Un résultat qui va dans le sens de quelques recherches antérieures. Selon G Simard et al. (2005) ce résultat est expliqué par la nature d'information partagé dans les entreprises étudiées. Informations concernant essentiellement le niveau corporatif.

Les résultats obtenus témoignent que la justice procédurale dépend de l'organisation de travail (H1.2). À ce niveau le coefficient de régression $\beta$ est de 0,443 , de même cette relation est significative à $\mathrm{p}<0.005^{* * * *}$. Ainsi, l'organisation de travail contribue à l'explication de justice procédurale avec un niveau de détermination de 22,2\%.

A travers notre recherche, nous avons pu identifier les pratiques d'organisation du travail comme étant des déterminants de la justice procédurale. Donc l'hypothèse mettant en relation l'organisation de travail et la justice procédurale est validée. 
Les managers qui cherchent à améliorer la perception de la justice procédurale peuvent jouer sur quatre pratiques en matière d'organisation du travail, à savoir :

L'élargissement des tâches via le regroupement « horizontal » de plusieurs tâches de même niveau de complexité afin de former un ensemble. Comme l'indique Nicolas (2014), en plus de la diminution de la pénibilité physique, cette pratique introduit une variété qui concourt à limiter la charge mentale. En effet, chaque salarié exécute davantage d'opérations et a une vision plus globale de l'activité, ce qui rend le travail plus intéressant (Nicolas, 2014, p. 51).

Afin de rendre le travail plus attractif, les responsables peuvent travailler sur l'enrichissement des tâches en optant pour un regroupement vertical, via l'introduction des tâches d'un niveau de complexité plus élevé (contrôles qualité, maintenance, réglages...). Cette pratique permet sans doute de responsabiliser les salariés, de favoriser la prise d'initiatives et d'augmenter d'avantage la motivation (Nicolas, 2014).

Comme le souligne Nicolas, (2014), ces deux pratiques d'élargissement et d'enrichissement des tâches peuvent être menées dans une logique complémentaire afin de rendre le travail à la fois moins répétitif et plus attractif.

Une troisième pratique dédiée à l'organisation de travail a été identifié comme importante, à savoir le développement de la polyvalence via la rotation. Ainsi, les salariés changent de poste selon un ordre cyclique et préétabli, à travers «la mise en place d'une organisation précise avec des changements prévus par exemple toutes les heures, à chaque pause ou par demi-journées. Cela permet d'alterner les différentes charges de travail : physique et mentale, ou physique/statique et physique/dynamique notamment. Par exemple, le long d'une chaîne de production, on trouvera intérêt à faire alterner les postes de surveillance visuelle et les postes d'alimentation qui supposent de soulever des charges lourdes » (Nicolas, 2014, p. 50).

Une dernière pratique susceptible d'améliorer le niveau de perception de la justice procédurale est celle liée à la constitution de groupe de résolution des problèmes. Les professionnels évoquent un autre concept lié à la mise en place de groupe semi-autonome qui consiste à laisser les individus organiser leur activité de travail afin d'atteindre un objectif.

Comme les suggère Nicolas, (2014, p. 53) « laisser les individus décider de leur organisation de travail est très motivant et permet de plus de les impliquer davantage dans la réflexion sur la sécurité et l'amélioration des conditions de travail ».

En résumé, l'amélioration de la perception de la justice procédurale passe par l'implantation de ces quatre pratiques exemplaires en matière de l'organisation de travail. 
La rémunération incitative a un effet direct et positif sur la justice procédurale H1.3, avec $\beta=0,266$. Cette relation est significative à $\mathrm{p}=0,021^{*}$ et $\mathrm{t}=2,303$. Cette variable contribue à l'explication de la justice avec un niveau de $18,6 \%$.

Sur la base des tests effectués, nous avons pu valider l'hypothèse selon laquelle la rémunération incitative influence directement et positivement la perception de la justice procédurale. Nos résultats corroborent ceux des études antérieures (Simard, Doucet, et Bernard 2005; Paré et Tremblay 2007; Ndayirata 2017; Tremblay, Michel, J. Cloutier, G. Simard, et D. Chênevert. 2005). La reconnaissance des efforts des employés par l'entreprise contribue à produire une perception favorable de justice.

Ainsi, plus la pratique de rémunération incitative est implantée plus la perception de la justice procédurale est positive. Cette justice peut prendre plusieursformes:

- Les salariés peuvent donner leurs opinions sur les procédures de l'entreprise.

- L'entreprise traite rapidement les demandes de formation des salariés.

- L'entreprise laisse toujours aux salariés le temps d'exprimer par rapport à leur travail.

Les tests effectués témoignent l'absence de relation entre la sécurité de l'emploi et la justice procédurale. Cette relation n'est pas significative (H1.4 $\beta=-0,210, t=1,171, p=0,077)$. Ainsi, cette quatrième sous hypothèse est rejetée.

En ce qui concerne le lien entre la formation et la justice procédurale (H1.5), les résultats obtenus affichent qu'il n'est pas significatif. Ainsi, cette cinquième sous hypothèse est rejetée. Le coefficient de détermination de la variable endogène « justice procédurale » est de 38,7\%.

Le test des hypothèses témoigne l'absence de relation entre l'implantation des programmes de formation et la perception de la justice procédurale. Les travaux de Chew et Chan (2008) ; Conway et Monks (2008) indiquent qu'il n'existe aucune relation significative. Selon ces chercheurs, les investissements en formation réalisés par l'entreprise favoriseraient le développement d'une perception positive. Par conséquent, l'association entre la pratique de formation et la justice procédurale aurait dû être significative et positive.

Toutefois, le résultat obtenu dans le cadre de cette étude ne permet pas de renforcer cette explication. Ce résultat va dans le même sens que celui de Meyer et Smith (2000) ainsi que Simard, Doucet, et Bernard (2005). D'après ces auteurs, il est possible que la formation doive être groupée à d'autres pratiques pour avoir un impact réel sur la performance $\mathrm{RH}$ par le biais de la justice. 
En conclusion, ces résultats confirment les constatations des recherches antérieures positionnant les pratiques de GRH comme antécédents de la justice procédurale (Simard, Doucet, et Bernard 2005; Charest 2005; Meyer et Smith 2000).

Les pratiques de GRH peuvent améliorer la performance RH, s'elles contribuent à développer le sentiment de justice. En effet, et selon Thibaut et Walker, (1975) cité par Tremblay, Michel, J. Cloutier, G. Simard, et D. Chênevert. 2005) «les procédures sont perçues d'autant plus justes lorsqu'elles accordent la possibilité d'exercer un contrôle, soit sur le processus décisionnel, soit sur les décisions menant aux résultats ».

Les tests effectués sur la base d'un modèle à équations structurelles en suivant l'approche PLS indiquent que la justice procédurale a un effet positif et significatif sur la performance RH (H2. $\left.\beta=0,350 ; t=2,872 ; p=0.005^{* *}\right)$. Donc, cette cinquième hypothèse est confirmée. Ainsi, la performance RH est déterminée à hauteur de 19,5\% .

Sur la base d'une lecture critique de la littérature, nous avons supposé que la perception de la justice procédurale influence directement et positivement le niveau de performance $\mathrm{RH}$.

Sur la base des tests effectués, nous avons pu valider cette hypothèse. Ces résultats se conjuguent parfaitement avec plusieurs travaux de recherche qui confirment qu'une perception positive de la justice organisationnelle conduit à l'amélioration du niveau de performance RH à travers l'amélioration du bien-être au travail (C. Piasecki\& Steiner, 2017 ; Provost Savard \& DagenaisDesmarais, 2018 ; Huong, Zheng, et Fujimoto 2016; Renaud, Tremblay, et Morin 2014; Müller et Djuatio 2011 ; M Tremblay, J Cloutier, G Simard 2010).

La perception de la justice représente un levier d'actions pour booster la performance individuelle (Zapata-Phelan, Cindy P., et al., 2009 ; Frimousse, Peretti\&Swalhi 2008).) et celle liée au volet RH (Caroline Piasecki, 2017). Ce qui est cohérent avec des recherches antérieures (Huong, Zheng, et Fujimoto2016; Renaud, Tremblay, et Morin 2014; Müller et Djuatio 2011).. En somme, il s'avère que la justice procédurale dépond des deux catégories des pratiques de GRH, à savoir : l'organisation de travail et la rémunération incitative. En plus, la justice procédurale contribue à l'explication de la performance $\mathrm{RH}$. 


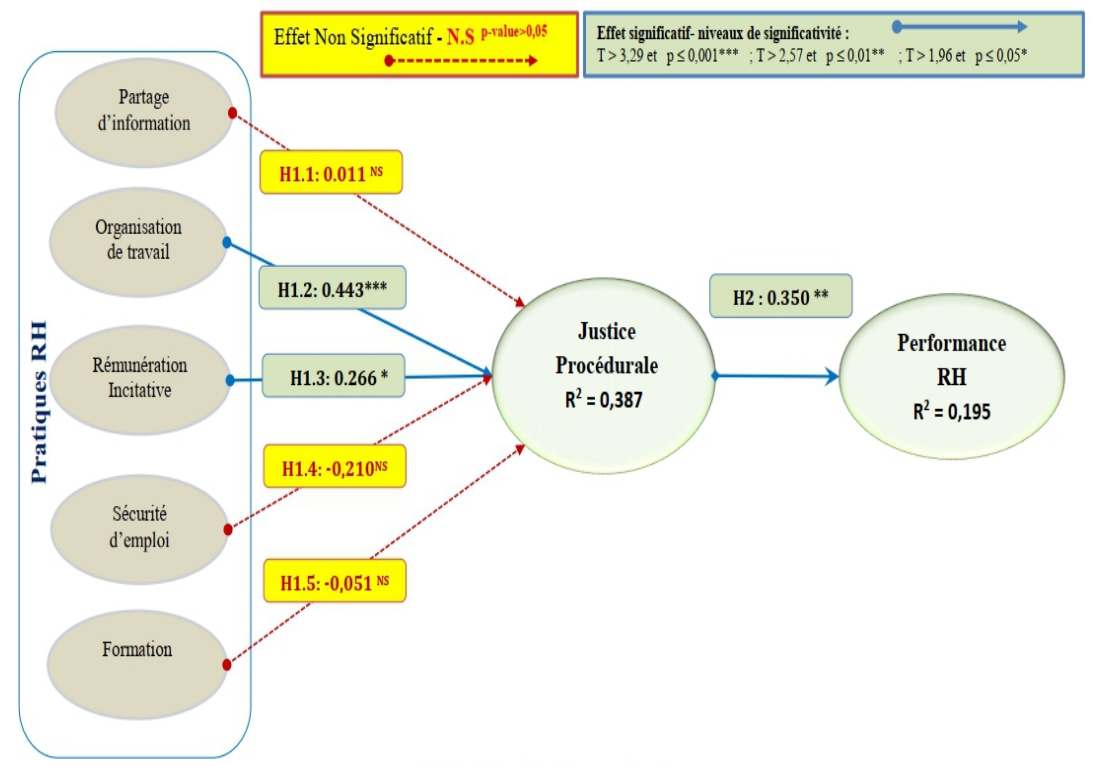

Figure 3. Modèle final de recherche estimé par les MES-PLS

La justice constitue l'un des éléments-clés permettant d'expliquer l'influence des pratiques de GRH sur les attitudes et les comportements en milieu de travail (Cloutier Julie, 2003 ; M Tremblay, J Cloutier, G Simard, 2010).

À la lumière de ces résultats, notre recherche a contribué autant sur le plan théorique que empirique. D'une part, elle a participé à l'enrichissement des connaissances en contribuant à la compréhension de « la boîte noire » qui lie les pratiques de GRH et les indicateurs de la performance. Et ce en proposant un modèle intégrateur (Pratiques de GRH - Justice procéduralePerformance RH). D'autre part, les managers des entreprises de l'industrie automobile peuvent opter pour ce modèle développé afin d'améliorer le niveau performance RH, en optant pour l'amélioration du niveau de perception de la justice procédurale via l'instauration des pratiques de GRH liées à l'organisation de travail et la rémunération incitative. En effet, les entreprises doivent se soucier de la perception de la justice s'elles veulent améliorer d'une manière significative le niveau des indicateurs de la performance $\mathrm{RH}$ par l'implantation des pratiques de GRH appropriées.

À notre question de recherche visant à vérifier si le fait d'implanter les pratiques degestion des ressources humaines peut améliorer et booster la performance RH des entreprises de l'industrie automobile via la justice procédurale, nous pouvons répondre par l'affirmative.

L'ensemble des résultats obtenus permettent de proposer un modèle facile à opérationnaliser dans le contexte marocain, afin de prendre des 
décisions permettant d'améliorer le niveau de performance RH en optant pour plusieurs interactions.

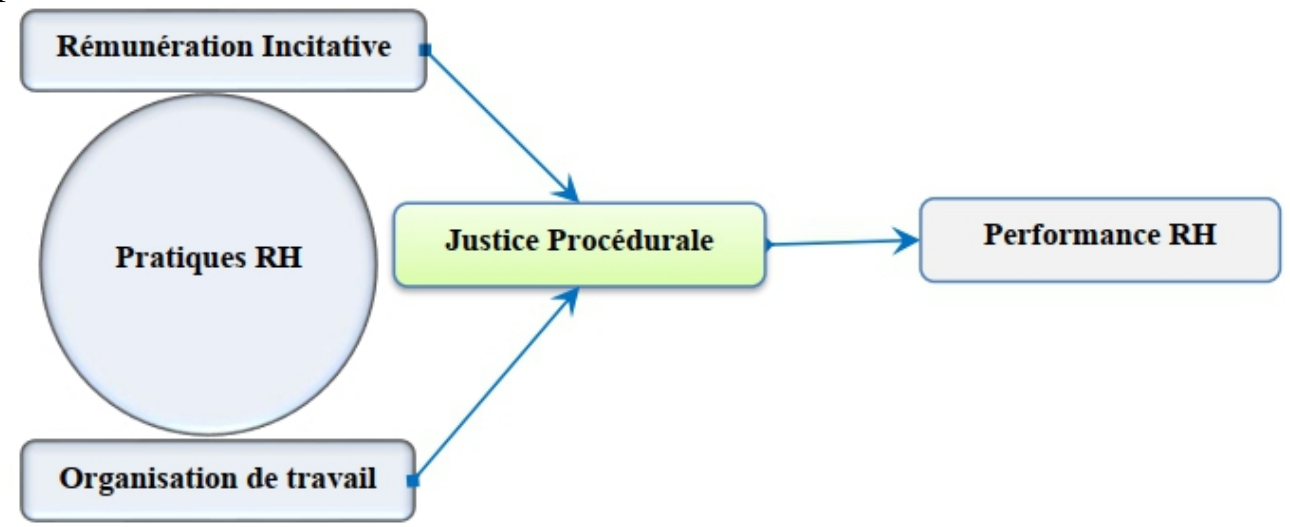

Figure 4. Le modèle de recherche validé

\section{Conclusion}

La présente étude s'inscrit dans un champ d'études en pleine expansion qui vise à appréhender et comprendre les liens GRH-Performance. Elle avait pour objectif d'étudier les modalités d'influence des pratiques de GRH sur la performance $\mathrm{RH}$, en mettant en question la place de la justice procédurale.

À la lumière des différents résultats obtenus, nous avons proposé un nouveau modèle facile à opérationnaliser dans le contexte marocain, Et ce afin de prendre des décisions permettant d'améliorer le niveau de performance $\mathrm{RH}$ des entreprises de l'industrie automobile au Maroc.

Les résultats de la présente recherche montrent qu'une gestion bien réfléchie des ressources humaines axée sur la rémunération incitative et l'organisation du travail peut contribuer à l'amélioration du niveau de performance RH. Le modèle développé souligne que l'augmentation de la performance RH passe nécessairement par l'amélioration de la perception de la justice procédurale via la prise en compte des pratiques de GRH. Nos résultats indiquent que ces pratiques ne peuvent influencer la performance RH que dans la mesure où elles sont perçues comme étant justes sur le plan procédural.

Notre recherche, comme c'est le cas pour les recherches en management des ressources humaines, comporte des limites :

La première limite est liée au modèle proposé. L'élaboration de notre modèle de recherche s'est basée que sur l'état de l'art, sans opter pour une approche de type qualitative exploratoire.

La deuxième limite renvoie à la validité externe qui détermine les possibilités de généralisation des résultats d'une recherche (Charreire Petit \& Durieux, 2014). À ce niveau, nous pouvons souligner cette limite, car notre recherche est mise en place en prenant en compte un seul secteur d'activité à savoir, le 
secteur de l'industrie automobile. A ce niveau, il serait intéressant de vérifier si l'on obtient les mêmes résultats en testant notre modèle dans d'autres secteurs d'activités.

En conclusion, Les résultats de notre recherche confirment que toute entreprise peut s'appuier sur plusieurs piliers, dans sa gestion des ressources humaines, dans le but d'améliorer sa performance. En effet, les entreprises doivent innover dans le domaine de GRH en instaurant des pratiques qui favorisent l'amélioration de la performance RH. Les managers ont tous intérêt à encourager l'implantation des pratiques de GRH et agir avec justesse afin d'améliorer la performance RH de leurs entreprises.

\section{References:}

1. AïtRazouk, A. (2007). Gestion stratégique des ressources humaines: recherche théorique et empirique sur la durabilité de la relation entre stratégie RH et performance (Doctoral dissertation, Nancy 2).

2. Aliouat, B., \&Besbes, A. (2013). Apprentissageorganisationnel, capacitéd'innover et Pratiques RH: Quels Impacts combinatoires sur la compétitivité et la performance? (No. halshs-00854677).

3. AllaniSoltan, N., Arcand, M., \&Bayad, M. (2005). Impact de la gestion stratégique des ressources humaines sur l'innovation. In Gestion internationale et pays emergents.

4. Ambrose, M. L., \&Schminke, M. (2009). The role of overall justice judgments in organizational justice research: A test of mediation. Journal of applied psychology, 94(2), 491.

5. Arcand, G. (2006). Étude du rôle de la culture nationale dans la relation entre les pratiques de GRH et de la performance organisationnelle: le cas des banques de vingt-deux pays d'Amérique du Nord, d'Europe et d'Asie (Doctoral dissertation).

6. Arcand, M. (2001). L'effet des pratiques de gestion des ressources humaines sur l'efficacité des caissespopulaires Desjardins du Québec (Doctoral dissertation, Université Paul Verlaine-Metz).

7. Arcand, M., Bayad, M., \&Fabi, B. (2002). L'effet des pratiques de gestion des ressources humaines sur l'efficacitéorganisationnelle des coopérativesfinancièrescanadiennes. Annals of public and Cooperative Economics, 73(2), 215-240.

8. Audet, M., Bélanger, L., Long, R. J., \&Galambaud, B. (1994). La gestion des ressources humaines: du modèle traditionnel au modèle renouvelé. Relations Industrielles/Industrial Relations, 49(1), 168187.

9. Becker, B. E., \&Huselid, M. A. (2006). Strategic human resources management: where do we go from here?. Journal of management, 32(6), 898-925. 
10. Boselie, P., Dietz, G., \& Boon, C. (2005). Commonalities and contradictions in HRM and performance research. Human resource management journal, 15(3), 67-94.

11. Boulianne, J. D. E. (2002). Vers une validation du construit performance organisationnelle (French text).

12. Boubker, O. (2017). Évaluation de la contribution des systèmes d'information à la performance et à la maturité des processus logistiques : Cas des entreprises de l'industrie automobile et aéronautique au Maroc. (Thèse de doctorat en Sciences de Gestion. Université Abdelmalek Essaadi, ENCG de Tanger).

13. Boubker, O., \& Chafik, K. (2016). Comprendre le rôle des systèmes d'information dans l'amélioration de la performance et la maturité des processuslogistiques: étude qualitative auprès de six entreprises de l'industrie automobile et aéronautique au Maroc. European Scientific Journal, ESJ, 12(28), 397-429.

14. Brien-Robidoux, E., Labrèche, C., Hatier, D. E., Paiement, A. M., \&Blais, M. La justice organisationnelle et l'épuisementprofessionnel: une nouvelle mesureselon les opportunités en milieu de travail.

15. Carrière, J., \& Barrette, J. (2005). Gestion des ressources humaines et performance de la firme à capital intellectuelélevé: une application des perspectives de contingence et de configuration. Canadian Journal of Administrative Sciences/Revue Canadienne des Sciences de l'Administration, 22(4), 302-315.

16. Charest, Ariane. (2005). L'impact des modifications des pratiques et des politiques de GRH sur l'engagement affectif des employés. PhD Thesis.

17. Chew, J., \& Chan, C. C. (2008). Human resource practices, organizational commitment and intention to stay. International journal of manpower, 29(6), 503-522.

18. Chrétien, L., Arcand, G., Tellier, G., \& Arcand, M. (2005). Impacts des pratiques de gestion des ressources humaines sur la performance organisationnelle des entreprises de gestion de projets. Revue internationale sur le travail et la société, 3(1), 107-128.

19. Cohen-Charash, Y., \& Spector, P. E. (2001). The role of justice in organizations: A meta-analysis. Organizational behavior and human decision processes, 86(2), 278-321.

20. Colquitt, J. A. (2001). On the dimensionality of organizational justice: A construct validation of a measure. Journal of applied psychology, 86(3), 386.

21. Conway, E., \& Monks, K. (2008). HR practices and commitment to change: an employee-level analysis. Human Resource Management Journal, 18(1), 72-89. 
22. Cropanzano, R., \& Byrne, Z. S. (2000). Workplace justice and the dilemma of organizational citizenship.

23. Cropanzano, R., \& Greenberg, J. (1997). Progress in organizational justice: Tunneling through the maze. International review of industrial and organizational psychology, 12, 317-372.

24. Delery, J. E., \& Doty, D. H. (1996). Modes of theorizing in strategic human resource management: Tests of universalistic, contingency, and configurational performance predictions. Academy of management Journal, 39(4), 802-835.

25. Besseyre Des Horts, C. H. (2004). La fonction RH, une fonctionstratégique: discoursouréalité?. HAL.

26. Duguay, D. (2006). Pratiques de gestion des ressources humaines, organisation du travail et mobilisation des employés: le rôle de la justice, du soutien et de la confiance (Doctoral dissertation, Université du Québec à Montréal).

27. El Adraoui, H. (2015). Effets de la GRH sur la performance: validation du modèle configurationnel auprès d'un échantillon de 92 entreprises au Maroc. Question (s) de management, (3), 43-56.

28. Folger, R., \&Konovsky, M. A. (1989). Effects of procedural and distributive justice on reactions to pay raise decisions. Academy of Management journal, 32(1), 115-130.

29. Gagnon, O., \& Arcand, G. (2012). L'augmentation de la performance organisationnelle par l'application de pratiques de GRH alignées à la stratégied'affaires.

30. Gangloff, B., Finkelstein, R.,\&Holmick, E. (2017). Justice organisationnelle et croyance en un monde juste: perceptions et réactions des dirigeantsd'entreprise vis-à-vis de leurs managers (in) justes. Revue Européenne de PsychologieAppliquée, 67(6), 327-337.

31. Gilbert, P., \& Charpentier, M. (2004). Comment évaluer la performance RH? Question universelle, réponsescontingentes. Revue de gestion des ressources humaines, 53(8-9), 29-42.

32. Gilbert, P., \& Charpentier, M. (2004). Comment évaluer la performance RH? Question universelle, réponsescontingentes. Revue de gestion des ressources humaines, 53(8-9), 29-42.

33. Huong, L., Zheng, C., \& Fujimoto, Y. (2016). Inclusion, organisational justice and employee well-being. International Journal of Manpower, 37(6), 945-964.

34. Huselid, M. A. (1995). The impact of human resource management practices on turnover, productivity, and corporate financial performance. Academy of management journal, 38(3), 635-672.

35. Imbert, J. (2007). Les tableaux de bord RH: construire, mettre en oeuvre et évaluer le système de pilotage. Editions Eyrolles. 
36. Kivimäki, M., Vahtera, J., Elovainio, M., Virtanen, M., \& Siegrist, J. (2007). Effort-reward imbalance, procedural injustice and relational injustice as psychosocial predictors of health: complementary or redundant models?. Occupational and environmental medicine, 64(10), 659-665.

37. Laaboubi, M. (2012). Contribution à l'étude de l'effet des pratiques de mobilisation des ressources humaines sur la performance des entreprises: Cas des entreprises de la régionSouss-Massa-Draâ.

38. Lambert, E. G., Hogan, N. L., Jiang, S., Elechi, O. O., Benjamin, B., Morris, A., ... \& Dupuy, P. (2010). The relationship among distributive and procedural justice and correctional life satisfaction, burnout, and turnover intent: An exploratory study. Journal of Criminal justice, 38(1), 7-16.

39. Louart, P. (1996). Enjeux et mesures d'une GRH performante. AM Fericelli et B. Sire.

40. Masterson, S. S., Lewis, K., Goldman, B. M., \& Taylor, M. S. (2000). Integrating justice and social exchange: The differing effects of fair procedures and treatment on work relationships. Academy of Management journal, 43(4), 738-748.

41. Meyer, J. P., \& Smith, C. A. (2000). HRM practices and organizational commitment: Test of a mediation model. Canadian Journal of Administrative Sciences/Revue canadienne des sciences de l'administration, 17(4), 319-331.

42. Beaudin, G., \&Savoie, A. (1994). L'efficacité de l'organisation: théories, représentations et mesures. Boucherville, Québec: G. Morin.

43. Müller, J., \&Djuatio, E. (2011). Les relations entre la justice organisationnelle, l'employabilité, la satisfaction et l'engagementorganisationnel des salariés. Revue de gestion des ressources humaines, (4), 46-62.

44. Naro, G. (2006). Les indicateurssociaux: du contrôle de gestion aux développementsrécents du pilotage et du reporting. Management et gestion des ressources humaines: stratégies, acteurs et pratiques, 43 .

45. Ndao, Assane. (2012) «Contribution de la GRH à la réalisation de la motivation au travail : validation de l'approche universaliste ». AGRH. https://www.agrh.fr/assets/actes/2012-ndao2.pdf.

46. Ndayirata, S. (2017). L'influence des perceptions des pratiques de GRH et des facteurs de contexteorganisationnel sur la fidélité des employés: cas des médecins du secteur public au Burundi (Doctoral dissertation, Université Paul Valéry-Montpellier III).

47. Ndayirata, S., Belghiti-Mahut, S., \&Briole, A. (2018). L'influence des perceptions des pratiques de GRH et de facteurs de contexteorganisationnel sur l'intention de départvolontaire: Cas des 
médecins du secteur public au Burundi. Journal de gestion et d'economiemedicales, 36(1), 61-83.

48. Paré, G., \& Tremblay, M. (2007). The influence of high-involvement human resources practices, procedural justice, organizational commitment, and citizenship behaviors on information technology professionals' turnover intentions. Group \& Organization Management, 32(3), 326-357.

49. Peretti, J. M. (2017). Ressources humaines. Vuibert.

50. Pesqueux, Y. (2004, December). La notion de performance globale.

51. Piasecki, C. (2017). L'utilisation de la justice organisationnelle dans une démarche de prévention des risquespsychosociaux (Doctoral dissertation, Côte d'Azur).

52. Pigeyre, F. (2006). Les modèles d'analyse de la GRH. Management et gestion des ressources humaines: stratégies, acteurs et pratiques, 7 .

53. Pottiez, J. (2011). Évaluation de la performance de la formation en entreprise par une approchesystémique (Doctoral dissertation, Lille 1).

54. Reb, J., Goldman, B. M., Kray, L. J., \&Cropanzano, R. (2006). Different wrongs, different remedies? Reactions to organizational remedies after procedural and interactional injustice. Personnel Psychology, 59(1), 31-64.

55. Renaud, S., Tremblay, F. A., \& Morin, L. (2014). L'impact de la justice organisationnelle sur la fidélisation: étude longitudinale auprès de travailleurs du secteur des TIC au Canada. Question (s) de management, (4), 11-26.

56. Salhi, M. (2016). Impact des discriminations sur le stress au travail: une mesure du rôle de la valorisationorganisationnelle (Doctoral dissertation, Grenoble Alpes).

57. Simard, G., Doucet, O., \& Bernard, S. (2005). Pratiques en GRH et engagement des employés: le rôle de la justice. Relations industrielles/Industrial Relations, 60(2), 296-319.

58. Simen, S. F. (2017, June). Pratiques de GRH dans les très petites entreprises sénégalaises: pertinence d'une gestion qui concilie tradition et modernité.

59. Taïeb, J. P. (2016). Valoriser la performance RH: Un enjeu pour la productivité de l'entreprise. Dunod.

60. Tremblay, M., Cloutier, J., Simard, G., \&Chênevert, D. (2005). Les pratiques RH et la performance prescrite et hors rôle: vérification $\mathrm{du}$ rôle médiateur de la justice, du support, de la confiance et de l'engagementorganisationnel. In AGRH, 16ème Conférence,"(Re) concilierl'économique et le social", Paris Dauphiné (Vol. 15). 
61. Tyler, T. R., \&Blader, S. L. (2003). The group engagement model: Procedural justice, social identity, and cooperative behavior. Personality and social psychology review, 7(4), 349-361.

62. Wu, P. C., \& Chaturvedi, S. (2009). The role of procedural justice and power distance in the relationship between high performance work systems and employee attitudes: A multilevel perspective. Journal of management, 35(5), 1228-1247.

63. Youndt, M. A., \& Snell, S. A. (2004). Human resource configurations, intellectual capital, and organizational performance. Journal of managerial issues, 337-360.

64. Zgoulli-Swalhi, S. (2014). Employabilité et implication organisationnelle: quelles pratiques RH? (Doctoral dissertation). 